etoposide $(n=67)$, etoposide and cyclophosphamide $(n=11)$ or etoposide and busulfan $(n=1)$. At transplantation, $62 \%$ of patients $(n=49)$ were in first complete remission (CR1) and $38 \%(n=30)$ were beyond CR1, respectively. Estimated overall survival rates were 54\% and $29 \%$ for patients in CR1 or beyond CR1, respectively $(P=0.01)$. Event-free survival rates were $48 \%$ and $26 \%$ for these respective groups $(P=0.02)$. Nonrelapse mortality rates were higher for patients beyond CR1 than in CR1 ( $54 \%$ versus $31 \%$; $P=0.05$ ), but both groups had a similar incidence of relapse ( $28 \%$ versus $41 \%$, respectively; $P=0.28$ ). The median time to relapse was prolonged in individuals beyond CR1 compared with those in CR1 (12 months versus 9 months). Univariate analysis showed that overall and event-free survival were affected by white blood cell count at diagnosis, disease status at transplantation and grades $2-4$ acute graft-versus-host disease.

These results showed that fractionated total body irradiation and etoposide induction therapy conferred long-term remission in patients with $\mathrm{Ph}^{+}$ALL.

Original article Laport GG et al. (2008) Long-term remission of Philadelphia chromosome positive acute lymphoblastic leukemia after allogeneic hematopoietic cell transplantation from matched sibling donors: a 20-year experience with the fractionated total body irradiation etoposide regimen. Blood 112: 903-909

\section{KRAS mutations do not predict response to 5-fluorouracil chemotherapy}

The treatment of choice for advanced colorectal cancer is 5-fluorouracil, but combinations of
anti-EGFR therapies with fluoropyrimidine chemotherapy have shown promise. Since KRAS mutations strongly predict resistance to antibodies that target the EGFR, Etienne-Grimaldi et al. carried out a study to investigate whether KRAS mutations influence treatment outcome in patients with stage IV colorectal cancer who received 5-fluorouracil treatment.

These authors previously reported that mutations in KRAS and p53 were not associated with outcome in 56 patients treated with 5-fluorouracil. Their current study included 93 patients with stage IV colorectal cancer and unresectable liver metastases, who were treated with 5-fluorouracil and leucovorin. Tumor samples were assessed for mutations in codons 12 and 13 of KRAS, polymorphisms in codon 72 of $\mathrm{p} 53$, and additional tumor variables.

KRAS mutations in codons 12 and 13 were present in 36 of 93 metastases. Perfect concordance of KRAS mutations between primary tumor and liver metastases was observed in 16 of 48 samples with such mutations. The presence of KRAS mutations was not significantly associated with levels of p53, thymidylate synthase, folypolyglutamate synthetase, or dihydropyrimidine activity. The response rate in patients with mutated KRAS metastases was $44.4 \%$, compared with $32.1 \%$ in those with wild-type metastases. KRAS status had no influence on survival.

The authors conclude that KRAS mutational status only predicts response to the anti-EGFR element of combination therapies; tumors with KRAS mutations can still respond to 5-fluorouracil-based therapy.

Original article Etienne-Grimaldi MC et al. K-Ras mutations and treatment outcome in colorectal cancer patients receiving exclusive fluoropyrimidine therapy. Clin Cancer Res 14: 4830-4835 